\title{
Precision tests of the $J / \psi$ from full lattice QCD: mass, leptonic width and radiative decay rate to $\eta_{c}$
}

\section{T. H. Davies;, G. C. Donald, R. J. Dowdall, J. Koponen}

University of Glasgow

E-mail: Christine.Davies@glasgow.ac.uk

\section{E. Follana}

Universidad de Zaragoza

\section{K. Hornbostel}

Southern Methodist University

G. P. Lepage

Cornell University

C. McNeile

Universität Wuppertal

HPQCD collaboration

We show results from calculations in full lattice QCD of the mass, leptonic width and radiative decay rate to $\eta_{c}$ of the $J / \psi$ meson. These provide few $\%$ tests of QCD. Another (1.5\%) test comes from comparison of time-moments of the vector charmonium correlator with results derived from the experimental values of $R\left(e^{+} e^{-} \rightarrow\right.$ hadrons $)$ in the charm region.

Xth Quark Confinement and the Hadron Spectrum,

October 8-12, 2012

TUM Campus Garching, Munich, Germany

\footnotetext{
* Speaker.
} 

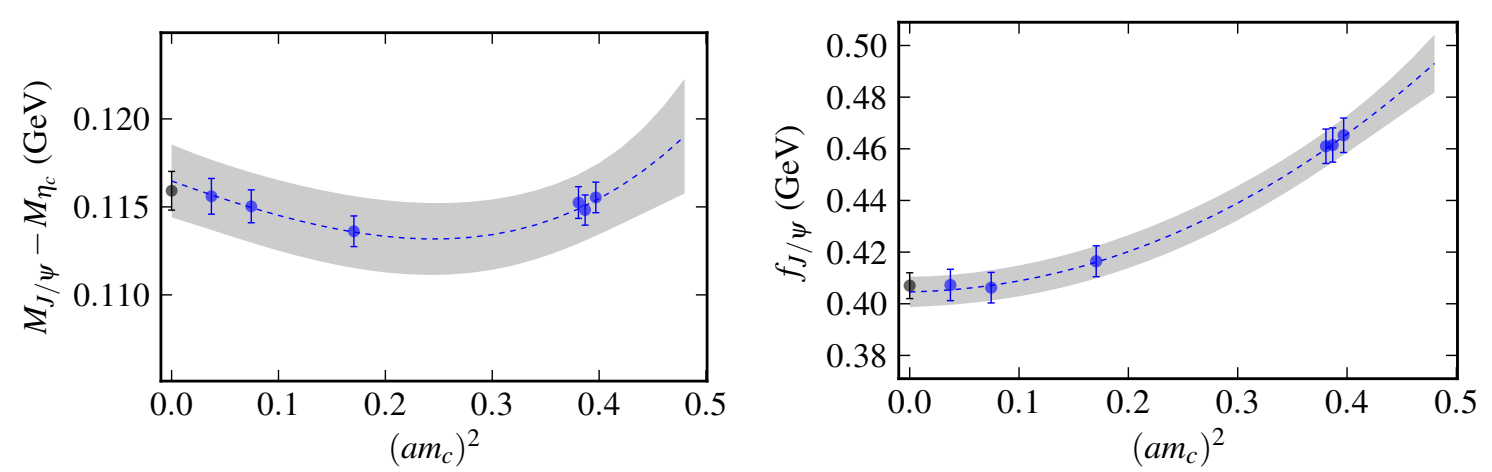

Figure 1: The charmonium hyperfine splitting on the left and decay constant of the $J / \psi$ on the right as a function of lattice spacing. The grey band is our fit to the calculated blue points. At $a=0$ it agrees well with the experimental value given by the black point.

\section{Introduction}

Precision tests of lattice QCD against experiment are critical to provide benchmarks against which to calibrate the reliability of predictions from lattice QCD [1]. Many of these tests are also important tests of QCD itself because frequently lattice QCD provides the only method for precision calculation. Most tests carried out so far have been based on the spectrum of gold-plated hadron masses. It is important to have tests that also involve decay matrix elements but, for weak decays, uncertainties in CKM elements are a limiting factor (indeed, the lattice calculations are used to determine the CKM elements). Electromagnetic decay rates therefore have the potential to provide good tests because their normalisation, related to $\alpha_{Q E D}$, is well-known.

Here we provide such tests for the $J / \psi$ including the full effect of $u, d$ and $s$ quarks in the sea for the first time. We are able to do this because we have developed a particularly accurate formalism for discretising the quark piece of the QCD Lagrangian onto a lattice, known as Highly Improved Staggered Quarks (HISQ) [2]. The work described here has now been published [3] and we refer to that paper for all details. Here we simply give highlights from the results. For a similar calculation using twisted mass quarks and including $u$ and $d$ quarks in the sea, see [4]. For earlier work in the quenched approximation see [5].

\section{Results}

Figure 1 shows results on the left for the hyperfine splitting $\left(M_{J / \psi}-M_{\eta_{c}}\right)$ and on the right for the $J / \psi$ decay constant, $f_{J / \psi}$, as a function of lattice spacing, $a$ (given in units of the $c$ quark mass, $m_{c}$ ). The blue points show our results at four different widely spaced values of $a$ and the grey band shows our fit, including the $1 \sigma$ error bar, which allows extrapolation to the real world at $a=0$. The black point at $a=0.0$ is the experimental value. For $f_{J / \psi}$ this is derived from $\Gamma\left(J / \psi \rightarrow e^{+} e^{-}\right)$.

Our value for the hyperfine splitting is $116.5(2.1)(2.4) \mathrm{MeV}$ where the first error is from our calculation and fit and the second is from uncertainties in the $\eta_{c}$ mass from electromagnetic effects and $\eta_{c}$ annihilation, neither of which are included in our lattice QCD calculation. The current 

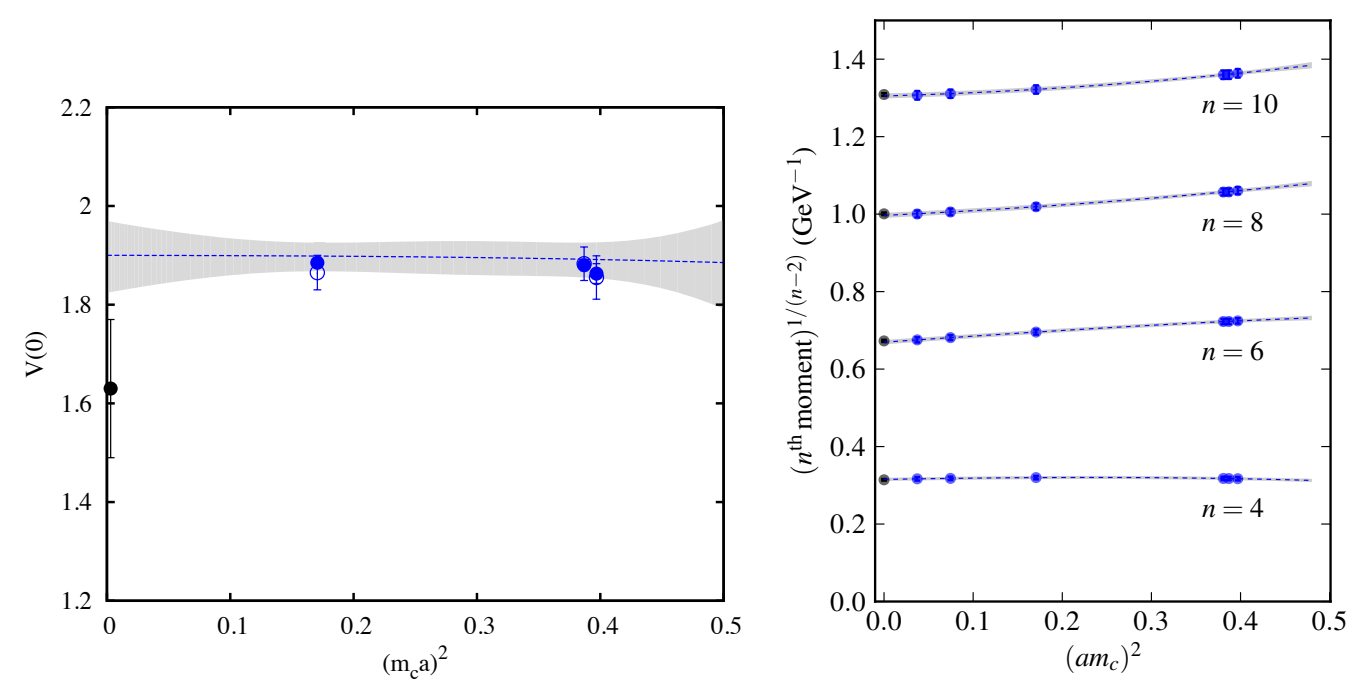

Figure 2: On the left is the value of the vector form factor between $J / \psi$ and $\eta_{c}$ at squared 4-momentum transfer equal to zero, and on the right the $n$th time-moments of the vector charmonium correlator for $n=4$, 6,8 , and 10. Symbols are as in Figure 1.

experimental average is $115.9(1.1) \mathrm{MeV}$ [6]. Our value for $f_{J / \psi}$ is $405(6)(2) \mathrm{MeV}$ to be compared to 407(5) MeV from experiment [6].

Figure 2 shows on the left results for the vector form factor at $q^{2}=0$ between the $J / \psi$ and $\eta_{c}$ which is related the decay rate for $J / \psi \rightarrow \gamma \eta_{c}$. Our final result for $\mathrm{V}(0)$ is 1.90(7)(1) giving $\Gamma\left(J / \psi \rightarrow e^{+} e^{-} \gamma \eta_{c}\right)=2.49(18)(7) \mathrm{keV}$. This is to be compared to the experimental value of 1.84(30) keV [6]. Agreement is acceptable, but an improvement in the experimental error would allow a much more stringent test of QCD.

The righthand plot of Figure 2 shows results for the 4th, 6th, 8th and 10th time-moments of the charmonium vector correlator. These moments test the short time behaviour of the correlator, where the large time behaviour gives the properties of the ground-state charmonium vector meson, the $J / \psi$, discussed above. The continuum limit of the time-moments can be compared to $q^{2}$ derivative moments of the charm quark vacuum polarisation function derived from experimental results for $R_{e^{+} e^{-}}$[7]. Good agreement with the experimental results is seen with errors at $1.5 \%$.

\section{References}

[1] C. Davies et al (HPQCD, UKQCD, MILC, Fermilab Lattice Collaborations), Phys. Rev. Lett. 92:022001 (2004), hep-lat/0304004.

[2] E. Follana et al (HPQCD Collaboration), Phys. Rev. D75:054502 (2007), hep-lat/0610092.

[3] G. C. Donald et al (HPQCD Collaboration), Phys. Rev. D86:094501 (2012), arXiv:1208.2855.

[4] D. Becirevic and F. Sanfilipo, arXiv: 1206.1445; F. Sanfilippo, these Proceedings.

[5] J. J. Dudek, R. G. Edwards and D. G. Richards, Phys. Rev. D73:074507 (2006), hep-ph/0601137.

[6] J. Beringer et al (Particle Data Group), Phys. Rev. D86:010001 (2012).

[7] J. H. Kuhn, M. Steinhauser and C. Sturm, Nucl. Phys. B778:192 (2007), hep-ph/0702103. 